\title{
First record of the genus Notiomys Thomas 1890 (Rodentia, Cricetidae) for Chile
}

\author{
Guillermo D’Elía ${ }^{1^{*}}$, Roderich Barria ${ }^{2}$ y Pablo Teta ${ }^{3}$
}

\footnotetext{
1 Instituto de Ciencias Ambientales y Evolutivas, Universidad Austral de Chile, campus Isla Teja s/n. Valdivia, Chile. E-mail: guille.delia@gmail.com (G'E).

${ }_{2}^{2}$ Ovejero 803-B Puerto Natales. Magallanes, Chile. E-mail: rorybarria@gmail.com (RB).

${ }^{3}$ Colección Nacional de Mastozoología, Museo Argentino de Ciencias Naturales Bernardino Rivadavia, Avenida Ángel Gallardo 470, C1405DJR. Buenos Aires, Argentina. E-mail: antheca@yahoo.com.ar (PT).

${ }^{*}$ Corresponding author
}

\begin{abstract}
The Patagonian assemblage of small mammals has long fascinated naturalists. Even when it is moderately diverse, several of its components are endemic to the region. One of these is the monotypic genus Notiomys, whose single species $N$. edwardsii is widely distributed in the steppes of Argentinean Patagonia. A dead mouse found in Parque Nacional Torres del Paine, Última Esperanza, Chile, which was determined as belonging to the species N. edwardsii. The just mentioned finding allows us to report the genus Notiomys for the first time to Chile. This result highlights the need of keeping doing field prospections even in areas whose mammal assemblages are presumably well characterized.

El ensamble patagónico de pequeños mamíferos ha fascinado desde siempre a los naturalistas. Aun cuando es moderadamente diverso, varios de sus componentes son endémicos de la región. Uno de éstos es el género monotípico Notiomys cuya única especie, $N$. edwardsii, es ampliamente distribuida en la estepa patagónica argentina. Un ratón muerto fue encontrado en el Parque Nacional Torres del Paine, Última Esperanza, Chile, que fue determinado como pertenecientemente a la especie N. edwardsii. El registro recién mencionado permite reportar el género Notiomys por primera vez para Chile. Este resultado resalta la necesidad de continuar con las colectas de ejemplares en campo, aún en áreas donde los ensambles de mamíferos están, presuntamente, bien caracterizados.
\end{abstract}

Key words: abrotrichini, Chelemys, Geoxus, Patagonia, Sigmodontinae.

๑ 2016 Asociación Mexicana de Mastozoología, www.mastozoologiamexicana.org

\section{Introducción}

El ensamble de roedores patagónicos ha llamado la atención de múltiples naturalistas. Incluso Darwin (1839), en su paso por la región, reconoció la abundancia y diversidad de este grupo de animales en la porción más austral de América del Sur, indicándola como una de las mayores del mundo. Si bien esta afirmación no necesariamente se ajusta con la realidad, los roedores patagónicos constituyen un ensamble relativamente diverso con varias especies endémicas a la región (Pardiñas et al. 2011; Lessa et al. 2012). Entra estas últimas se encuentra el roedor fosorial Notiomys edwardsii (Thomas, 1890), única especie reconocida de Notiomys Thomas 1890. Éste género forma parte de la tribu Abrotrichini, cuya extensión geográfica va desde el Altiplano de Perú y Bolivia hasta Tierra del Fuego e islas adyacentes (D'Elia et al. 2015b).

Aun cuando Notiomys es raro en colecciones, este ratón presenta una amplia distribución en la Estepa Patagónica de Argentina, extendiéndose desde el sur de la provincia de Río Negro hasta el centro sur de la provincia de Santa Cruz (Andrade 2008; Pardiñas et al. 2008). A través de esta amplia distribución la especie ha sido registrada en estepas arbustivas y herbáceas desde la costa Atlántica de la provincia de Santa Cruz hasta altitudes de casi 1,600 m en la Meseta de Somuncurá en 
la provincia de Río Negro (Pardiñas et al. 2008). En esta nota damos a conocer el primer registro para Chile del género Notiomys.

\section{Materiales y Métodos}

El primer registro chileno de Notiomys se basa en un ejemplar hallado el 8 de abril de 2015 en el sector del Río Serrano, Parque Nacional Torres del Paine, Última Esperanza, Magallanes y Antártica Chilena ( $-51^{\circ} 11^{\prime} 29.36^{\prime \prime} \mathrm{S},-72^{\circ} 57^{\prime} 38.32^{\prime \prime} \mathrm{O}, \mathrm{ca} .30 \mathrm{~m}$; Figura 1). El ambiente donde el individuo fue encontrado corresponde a la Estepa Patagónica, compuesta fundamentalmente por gramíneas de altura media a baja, incluyendo Festuca sp. y Stipa sp.

\section{Resultados}

El animal base de este registro fue fotografiado (Figura 2) por uno de los autores (RB) pero no fue colectado. No obstante, la determinación del mismo a nivel genérico y específico es inequívoca de acuerdo a la siguiente combinación de caracteres que es exclusiva del género Notiomys (cf. Pardiñas et al. 2008; Teta 2013), incluyendo: cuerpo fusiforme, con miembros cortos; coloración dorsal castaña, con tintes anaranjados hacia los flancos y nítidamente delimitada del blancogrisáceo del vientre; manos muy peludas, dorsalmente cubiertas por pelos blancos y con garras desarrolladas; pies cortos y anchos, cubiertos por pelos cortos blancos y anaranjados, con un fleco de pelos blancos visible sobre el borde externo; cola bicolor; orejas muy pequeñas y ocultas entre los pelos de la cabeza; vibrisas mistaciales numerosas, con un mechón de pelos blancos que nace en la base de la oreja y la sobrepasa; ojos medianos; pelos alrededor del hocico anaranjado;

Figura 1. Registros de Notiomys edwardsii en el extremo austral de América del Sur. Los registros previamente conocidos se indican con círculos negros (tomados de Pardiñas et al. 2008; Tabla 1) y el primer registro para Chile es indicado con una estrella.

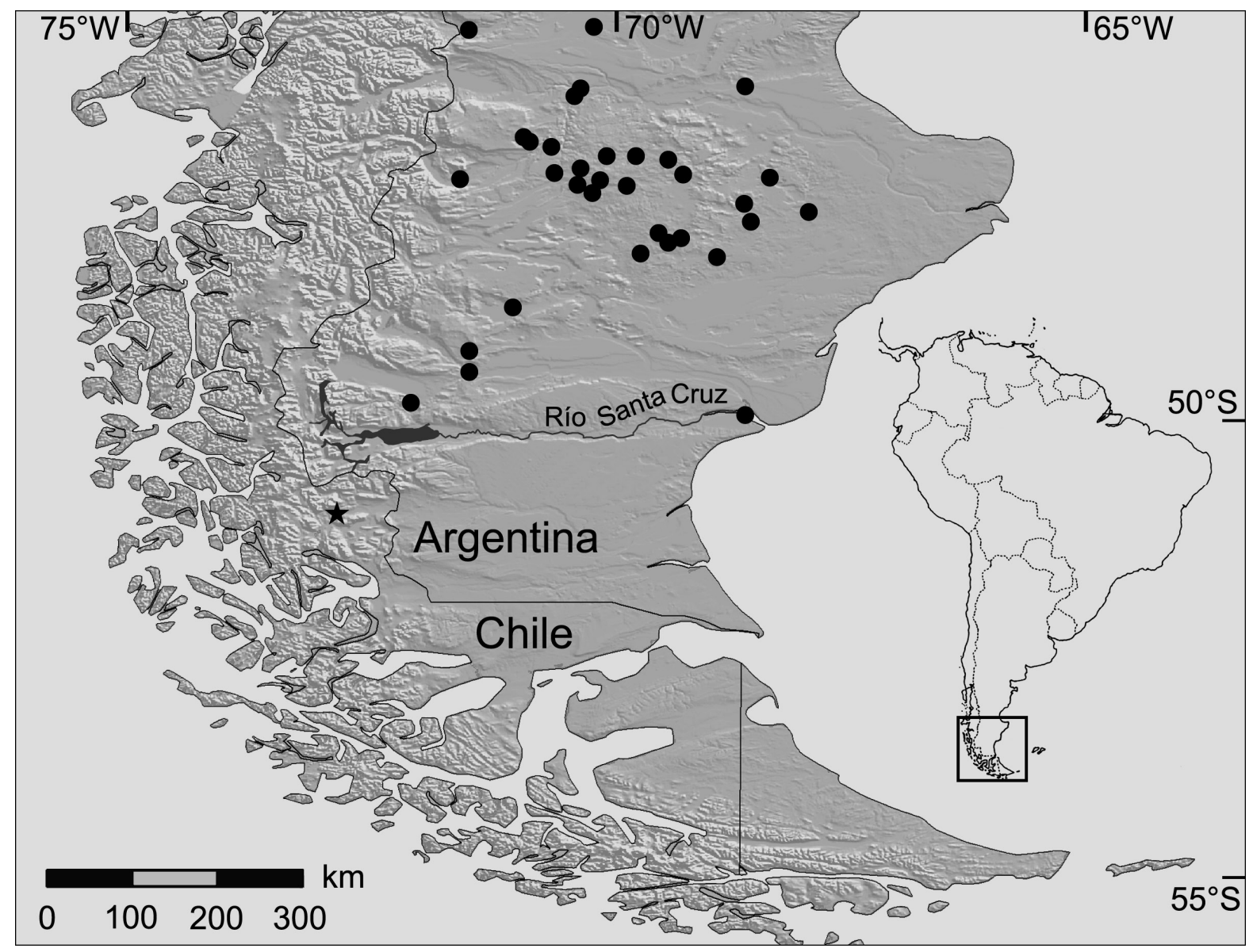


rinario rosado y conspicuo. La ausencia de escala en las fotografías impide hacerse una idea del tamaño, la observación directa del espécimen realizada el momento de fotografiarlo indica que se trataba de un animal pequeño, no mayor a 80 - $90 \mathrm{~mm}$ de cabeza-cuerpo. Otros abrotriquinos fosoriales, como Chelemys Thomas 1903 o Geoxus Thomas 1919, con los cuales podría confundirse, presentan una coloración general más oscura, con menos contraste entre el dorso y el vientre y sin tonos anaranjados, pabellones auriculares pequeños pero externamente visibles y carecen del fleco de pelos blancos sobre el borde externo del pie (cf. Teta 2013).

\section{Discusión y conclusiones}

Además de ser el primer registro del género Notiomys para Chile, el mismo constituye el más austral del género, ubicándose ca.160 km al sur del registro más cercano (Estancia La Ascensión, Santa Cruz, Argentina; Cueto et al. 2008; Figura 1). Es de interés notar que este es el primer registro del género al sur del Río Santa Cruz y del sistema de lagos del cual dicho río es efluente. De esta forma, el género Notiomys no es endémico de Argentina, pero sí de la Estepa Patagónica. Con este registro se aumenta a 29 el número de géneros de roedores conocidos en Chile.

Este nuevo registro muestra, una vez más, la necesidad de continuar con el trabajo de campo, aún de grupos considerados como bien conocidos como los mamíferos (D’Elía et al. 2015a) y/o áreas supuestamente bien conocidas como el sur de Chile (véase Osgood, 1943; Rau et al. 1978; Johnson et al. 1990). Para el Parque Nacional Torres del Paine, Johnson et al. (1990) han citado, pero sin indicar un ejemplar de referencia, a Chelemys delfini, otro ratón cavador de estatus incierto, pero que por sus características más probablemente se trate de un sinónimo de Geoxus michaelseni (Teta 2013; Teta et al. 2015a). Así, en el sur de Chile se registran por lo menos tres especies de ratones de

Figura 2. Vista externa de Notiomys edwardsii de Río Serrano, Parque Nacional Torres del Paine, Magallanes, Chile. Se destaca el contraste entre la coloración dorsal y ventral, la coloración anaranjada alrededor del hocico y en los flancos, las orejas pequeñas y escondidas en el pelaje de la cabeza y la cola corta (para otros caracteres, véase el texto).

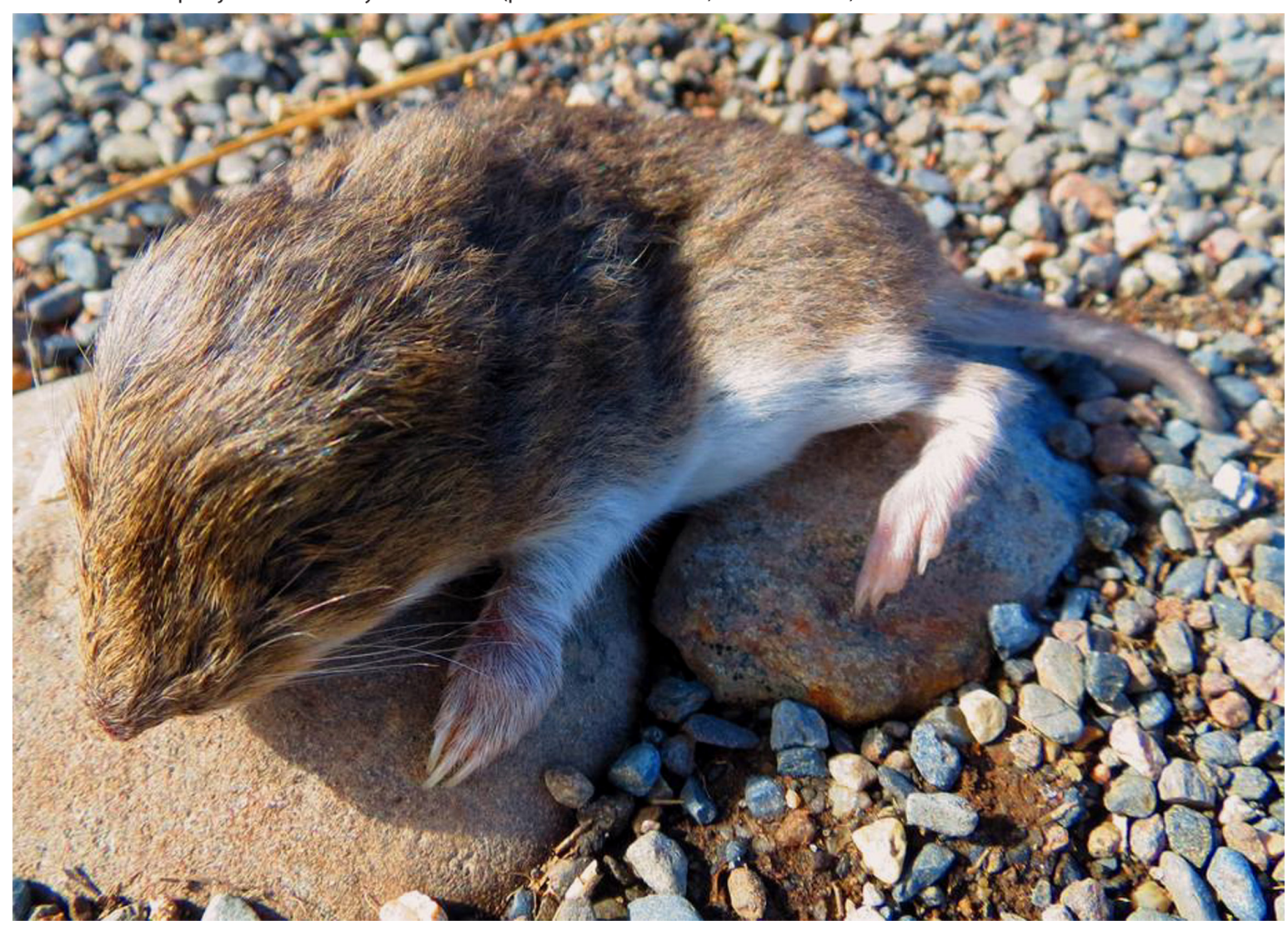


hábitos semifosoriales, incluyendo a Chelemys macronyx, Geoxus michaelseni y Notiomys edwardsii (Teta et al. 2015a, Teta et al. 2015b; este trabajo). De igual forma, todos los géneros de Abrotrichini, según hoy es entendida la tribu (Teta 2013; D’Elia et al. 2015b), están presentes en Chile.

En los últimos dos años, tres nuevas especies de roedores han sido reportadas para Chile. Dos de ellas, Eligmodontia dunaris y Abrothrix manni, constituyeron especies nuevas para la ciencia y fueron descritas con base en especímenes colectados en Chile (Spotorno et al. 2013, D’Elía et al. 2015a). Abrothrix jelskii, previamente conocida de Argentina, Bolivia y Perú fue recientemente registrada en el norte de Chile (Zuñiga y Tancara 2014). A esta lista sumamos a Notiomys edwardsii, previamente conocida de Argentina, que constituye también la primer mención del género para Chile. Valga esto último como refuerzo de la necesidad de continuar con el trabajo de colecta de mamíferos chilenos, aún en áreas consideradas bien exploradas como el extremo sur del país.

\section{Agradecimientos}

A dos revisores anónimos que realizaron sugerencias que mejoraron una versión anterior de este trabajo.

\section{Literatura citada}

Andrade, A. 2008. Mammalia, Rodentia, Cricetidae, Notiomys edwardsii (Thomas, 1890): Distribution extension and geographic distribution map. Check List 4:33-36.

Cueto, G. R., P. Teta, y P. De Carl. 2008. Rodents from southern Patagonian semi-arid steppes (Santa Cruz Province, Argentina). Journal of Arid Environments 72:56-71.

D’Elía, G., P. Teta, N. S. Upham, U. F. F. Pardiñas, y B. D. Patterson. 2015a. Description of a new softhaired mouse, genus Abrothrix (Sigmodontinae), from the temperate Valdivian rainforest. Journal of Mammalogy 96:839-853.

D’Elía, G., P. Teta, y U. F. F. Pardiñas. 2015b. Tribe Abrotrichini D'Elía, Pardiñas, Teta, and Patton, 2007. Pp. 107-109 en Mammals of South America, Vol. 2: Rodents (J. L. Patton, U. F. J. Pardiñas, y G. D’Elía, eds.). University of Chicago Press. Chicago, EE. UU.

Darwin , C. R. 1839. Narrative of the Surveying Voyages of His Majesty's Ships Adventure and Beagle Between the Years 1826 and 1836, Describing their Examination of the Southern Shores of South America, and the Beagle's Circumnavigation of the Globe. Journal and remarks. 1832-1836. Henry Colburn. London, England.

Johnson, W. E., W. L. Franklin, y J. A. Iriarte. 1990. The mammalian fauna of the northern Chilean Patagonia: a biogeographical dilemma. Mammalia 54:457-469.

Lessa, E. P., G. D’Elía G, y U. F. J. Pardiñas. 2012. Mammalian biogeography of Patagonia and Tierra del Fuego. Pp. 379-398 en Bones, clones, and biomes: an 80-million year history of Recent Neotropical mammals (Patterson, B. D., y L. P. Costa, eds.). University of Chicago Press. Chicago, EE. UU.

Osgood, W. H. 1943. The mammals of Chile. Fieldiana, Zoological Series 30:1-268.

Pardiñas, U. F. J., D. E. Udrizar Sauthier, P. Teta, y G. D’Elía. 2008. New data on the endemic Patagonian long-clawed mouse Notiomys edwardsii (Rodentia: Cricetidae). Mammalia, 72:273-285.

Pardiñas, U. F. J., P. Teta, G. D'Elía, y E. P. Lessa. 2011. The evolutionary history of sigmodontine rodents in Patagonia and Tierra del Fuego. Biological Journal of the Linnean Society 103:495-513.

Rau, J., J, Yáñez, Y F. Jaksic. 1978. Confirmación de Notiomys macronyx alleni O. y Eligmodontia typus typus C., y primer registro de Akodon (Abrothrix) lanosus T. (Rodentia: Cricetidae) en la zona de Última Esperanza (XII Región, Magallanes). Anales del Instituto de la Patagonia 9:203-204.

Spotorno A. E., C. Zuleta, L. Walker, G. Manríquez, P. Valladares, y J. C. Marín. 2013. A small, new gerbilmouse Eligmodontia (Rodentia: Cricetidae) from dunes at the coasts and deserts of north-central Chile: molecular, chromosomic and morphological analyses. Zootaxa 3683:377-394. 
TetA, P. 2013. Relaciones filogenéticas de la tribu Abrotrichini (Rodentia, Cricetidae): análisis separados y combinados de evidencias morfológicas y moleculares. Ph. D. dissertation, Universidad Nacional de La Plata. La Plata, Argentina.

Teta, P., U. F. J. Pardiñas, y G. D’Elía. 2015a. Genus Geoxus Thomas, 1919. Pp. 132-135 en Mammals of South America, Vol. 2: Rodents (J. L. Patton, U. F. J. Pardiñas, y G. D'Elía, eds.). University of Chicago Press. Chicago, EE. UU.

Teta, P., U. F. J. Pardiñas, y G. D’Elía. 2015b. Genus Chelemys Thomas, 1903. Pp. 127-132 en Mammals of South America, Vol. 2: Rodents (J. L. Patton, U. F. J. Pardiñas, y G. D'Elía, eds.). University of Chicago Press. Chicago, EE. UU.

Thомаs, O. 1890. Muridae. Pp. 1-32 en Mission scientifi que du Cap Horn, 1882- 1883. 6. Zoologie. Mammiferes (Milne- Edwards, A., ed.). Gauthier-Villars et Fils. Paris, Francia.

ZuÑIGA, E., Y R. TANCARA. 2014. Primer registro de Abrothrix jelskii (Thomas, 1894) (Mammalia: Rodentia: Cricetidae) en Chile. Biodiversidata 2:120.

Submited: November 4, 2015

Reviewed: February 19, 2016

Accepted: March 2, 2016

Associated editor: Sergio Solari 
